Research Article

\title{
Freemium of Digital Products with Small-World Network
}

\author{
Wei Li (D) \\ Research Center for Contemporary Commercial Services, School of Business, Hebei University of Economics and Business, \\ Shijiazhuang, China
}

Correspondence should be addressed to Wei Li; weilee@heuet.edu.cn

Received 24 April 2019; Revised 31 October 2019; Accepted 5 March 2020; Published 27 April 2020

Academic Editor: Ricardo Branco

Copyright (c) 2020 Wei Li. This is an open access article distributed under the Creative Commons Attribution License, which permits unrestricted use, distribution, and reproduction in any medium, provided the original work is properly cited.

"Freemium" is a popular business model adopted by the vendors of digital products, and it has aroused extensive attention in the academia. The existing research studies commonly explore the business model from the perspective of network effect, but lack the attention to the small-world features of network effect. In order to explore the effect of the small-world network, the current work presents a two-period optimization model of monopolist. The optimization model is incorporating with the Freemium model and the small-world feature of consumer base. The optimization model is solved analytically, and the comparative static results show that if the integrating network effect caused by the strong and weak relationship group is sufficiently high (or the small-world feature of the user group is prominent), the user group network exerts a positive effect; if the integrating network effect is not sufficiently high (or the small-world feature of the user group is not prominent), the user group network exerts a negative effect; especially, if the integrating network effect is low or moderate, the premium product is supposed to be free for the consumers. The conclusions enrich the understanding on the operation of digital products firms in the academia and industry.

\section{Introduction}

The business model of Freemium is named by Wilson [1] using the portmanteau word combining "free" and "premium." Anderson [2] describes this business model as offering a basic version for free to anyone in order to attract some users to pay for the upgrading premium version. Freemium is popular in the digital products industry: firms often allow consumers to use products with basic functionalities for free and charge for the products with valueadded functionalities [3]. For example, YouTube allows users to watch videos for free but charge for the value-added services such as avoiding advertisements. For another instance, mobile app developers provide free products and charge through in-app purchase $[4,5]$.

If done right, Freemium can create massive a user base for the companies and convert free users to paying customers [6]. Industry data shows that the $98 \%$ profit of Google's Play Store and 95\% profit of Apple's App Store are contributed by the Freemium applications [7]. However, in reality, many digital products firms still struggle with the consequences of this free-to-fee switch [8]. For example, the mobile app developers hesitate to charge the hidden prices through in-app purchase [9]. Therefore, how to determine the price of value-added products to maximize profit in this "trying before buying" service is a sharp challenge for digital products vendors when they implement the Freemium model.

In the digital products industry, the social network properties of the user base significantly impact the decisions of the firms [10]. It leads to the network externality effect that enables the users to enjoy a better utility from the installed base. The reason why the network externality improves consumers' utility is that the consumers could obtain additional utility depending on the size of the user base [11]. For example, the group-wide interactions help the individual users improve their understanding of product performance, which leads to a higher utility [12]. Hence, impacted by the network effect, consumers might be willing to pay a higher price for the product [13].

An important fact needed to be considered is that the social network, which exists in the user base and generates network externality, is not a regular network, but a smallworld network. In a regular network, each node is equally 
connected with the other nodes (see Figure 1). However, the consumer community in the reality often exhibits such a small-world feature: each member of a group has a close relationship with a few members, and these members' performance has greater impact than others (see Figure 2). The network with this feature is called a "small-world network" [14], which is popular in digital products. For example, in Twitter and Facebook, users have a strong interaction with a few closely related people or a few opinion leaders. Therefore, digital products vendors are supposed to determine the optimal pricing strategy with the small-world network.

To explore the optimal pricing of digital products with the small-world network externality, the current work develops a consumer utility model integrating the small-world network effect. Basing on the utility model, the current work also develops the optimization model on the pricing decision of digital products vendor. The decision model is solved mathematically, and the comparative statics show that the small-world feature of the user base may not contribute to a higher profit unless it is not prominent (or the intensity of network effect is high). In addition, if the intensity of network effect is low or moderate, the premium product is supposed to be adopted freely to enlarging the installed base. Comparing with the previous studies, the current work contributes a novel understanding that the small-world network has a dual effect on the Freemium model. This understanding serves as a useful supplement to the existing research, and enriches the knowledge on the role of network externality effects.

The rest of the paper is organized as follows. Section 2 reviews the literatures related to the current work. Section 3 explains the development of the utility function that incorporates the small-world network effect and the optimization model of the digital products vendor. Section 4 shows the solution of the optimization decision model and the comparative static analysis. The comparative static analysis is conducted for exploring the impact of such networks on the digital products' price and the vendor's profit. The conclusions and limitations of the current work are presented in Section 5.

\section{Literature Review}

Despite its increasing popularity, Freemium, the business model widely adopted by the digital products firms, still services as a topic of heated debate. For example, the firms always face the dilemma of balancing between improving the quality of the free product and creating demand and the value-added product: providing a free product with enough high quality may decrease the demand of the premium product for it may not offer significant added value [15].

The prior literatures exploring the Freemium model of digital products focus on the network externality effect, which is the prominent economic characteristic of digital products. Cheng et al. [16] explored a special free strategy called "the hybrid free strategy" that combines the characteristics of functionality- and time-limited free trial strategies. They

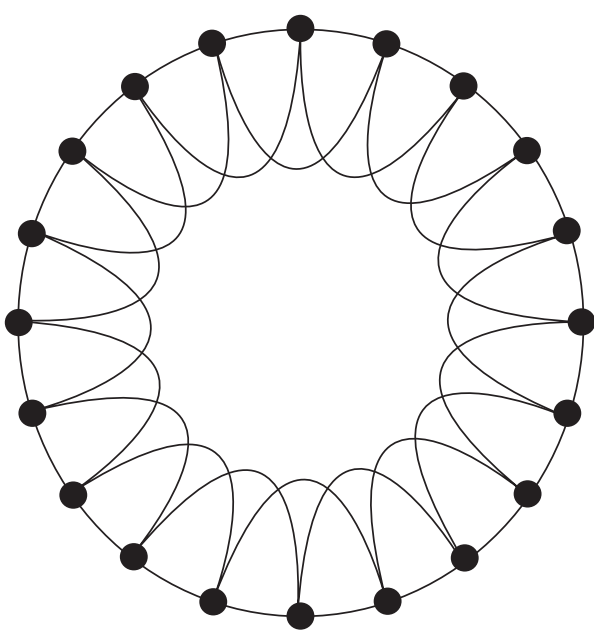

FIgURE 1: The regular network.

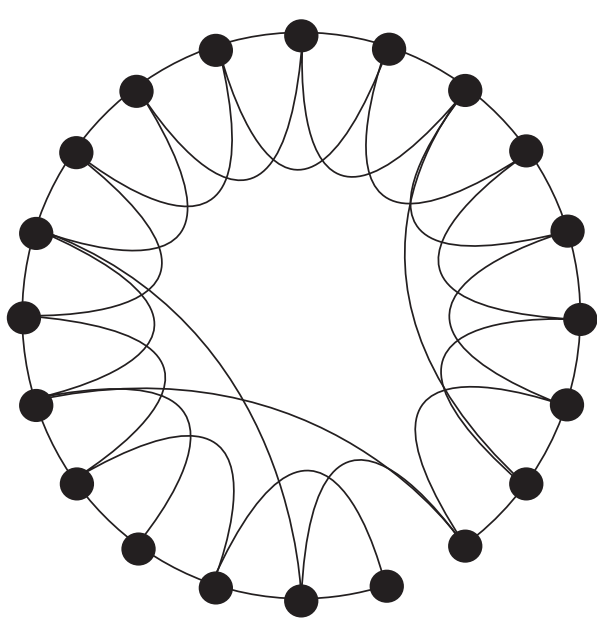

Figure 2: The small-world network.

explored the impact of network effect on the price and profit of the vendors in the hybrid free model. Shi et al. [17] investigated when Freemium is optimal for companies with network externality. They found that Freemium might be typically suboptimal even with the network effect, and the firm is supposed to charge the low-end product for free only if the consumers of the high-end product could obtain larger utility from an expansion of the installed base.

The prior literatures mentioned above are based on a potential hypothesis that the network formed by the consumer group is the regular network in which all members of the group pose the same impact on other member. In contrast with the existing literatures, the current work captures the small-world feature of the user base that the consumers would be impact more by their closed "friend." The small-world feature is very common in social network [18], but the existing research lacks analytical investigation on it. In addition, the prior literatures believe that the user base is positively impacting the products' prices and suppliers' profits; on the contrary, the current work suggests that taking into the small-world feature, the user base may also perform a negative effect. 


\section{Model Settings}

3.1. The Product's Behavior. A digital products provider $M$ with market monopoly launches free product $s_{F}$ and valueadded product $s_{P}$ pricing as $p$. The two products, $s_{F}$ and $s_{P}$, are compatible with each other; moreover, the functionalities of $s_{P}$ are better than $s_{F}$, i.e., $s_{P}>s_{F}$. As is known to all, digital products (e.g., Google Chrome and YouTube) definitely have some functionality; therefore, it is defined that $s_{F}>0, s_{P}>0$. Both of $s_{F}$ and $s_{P}$ are given exogenously, and $M$ determines the optimality of $p$ to maximize the profit.

3.2. Consumers' Utility. Following Hotelling's spatial model, the current work assumes that the consumer $v_{i}$ uniformly distributed over $[0,1]$, and the value of $v_{i}$ represents the valuation of the consumer. In the reality, consumers tend to experience free products first and then decide whether to purchase value-added products according to their own experience. Hence, a two-period model is presented in the current work to simulate the consumers' behavior. In the free-period, consumers decide whether to experience the free product $s_{F}$. In the premium-period, consumers who choose the free product $s_{F}$ decide whether or not to purchase the value-added product $s_{P}$. Each consumer will select at most one unit of $s_{F}$ or $s_{P}$.

3.2.1. Consumers' Utility from $s_{F}$. In the current work, $s_{F}$ represents the functionalities of the free product, and the subscript $F$ represents free-period. The utility of consumer $v_{i}$ obtained from free products $s_{F}$ in the free-period consists of two parts. The first part is the utility from the inherent value of $s_{F}$. This part is defined as $v_{i} \cdot s_{F}$. The second part is the utility from the effect of network externality. In the smallworld networks, the nodes have a closer relationship with a few other nodes. Therefore, parameter $\eta(0 \leq \eta \leq 1)$ is defined as the small-world coefficiency of the consumer base. $\eta$ represents the proportion of the individuals closely relating to a certain consumer in the group: when $\eta$ is small, the small-world feature of the consumer base is prominent (the consumers are closely related to only a few individuals); specially, if $\eta$ achieves the lower bound $(\eta=0)$, it means that the consumers have no closely related individuals in the user base. When $\eta$ is high, the small-world feature of the consumer base is weak (the consumers are closely connected with a large number of individuals in the user base); specially, if $\eta$ achieves the upper bound $(\eta=1)$, it means that all the consumers closely relate with each other in the user base.

The user base of $s_{F}$ is defined as $Q_{F}$; hence, $\eta \cdot Q_{F}$ and $(1-\eta) \cdot Q_{F}$ are, respectively, the number of individuals strong and weakly relating to the comsumer. Therefore, $\eta$. $Q_{F}$ could impose a strong network effect on consumers' utility, and $(1-\eta) \cdot Q_{F}$ imposes a weak network effect on consumers' utility. In the current work, the intensity of the network externalities generating from these two groups are $\lambda_{s}$ and $\lambda_{w}\left(\lambda_{s}>\lambda_{w}\right)$. The two parameters, respectively, measure the extent that each an additional user of the two relationship groups contributes to consumers' utility.
Therefore, the utility of $v_{i}$ obtained from $s_{F}$ is shown in equation (1), where $c_{F}$ is the learning cost of $s_{F}$.

$$
u_{F}\left(v_{i}, s_{F}\right)=v_{i} \cdot s_{F}+\lambda_{s} \cdot \eta \cdot Q_{F}+\lambda_{w} \cdot(1-\eta) \cdot Q_{F}-c_{F} .
$$

3.2.2. Consumers' Utility from $s_{P}$. In the current work, $s_{P}$ represents the functionalities of the premium product, and the subscript $P$ represents the premium-period. In the premium-period, $M$ introduces the value-added product $s_{P}$, and the utility of consumers obtained from $s_{P}$ consists of two parts. The first part is the utility from the functionalities of $s_{P}$, and it is denoted as $v_{i} \cdot s_{P}$ in the current work. The second part is the utility from the network effect generated by user base. Given the compatibility of $s_{F}$ and $s_{P}$, all the consumers who adopted $s_{F}$ would have network effect on the consumers who purchase $s_{P}$. Therefore, the network effect of $s_{P}$ is still generated by the user base $Q_{F}$. The intensity of network effect in the premium-period is assumed to be equal to that in the free-period. The reason is that the intensity of network effect depends on the characteristic of individual user. For example, if the consumers are capable of communicating with each other, they would enjoy a higher utility from the installed base, and vice versa. In reality, the characteristic of users are relatively stable with time; therefore, the intensity of network effect is stable during the two periods. Hence, the network effect of the strong relationship group of $v_{i}$ is $\lambda_{s}$. $\eta \cdot Q_{F}$ and that of the weak relationship group of $v_{i}$ is $\lambda_{w} \cdot(1-\eta) \cdot Q_{F}$. The learning cost of $s_{P}$ is defined as $c_{P}$, and the utility that consumer $v_{i}$ obtains from $s_{P}$ is shown in the following equation:

$$
u_{P}\left(v_{i}, s_{P}\right)=v_{i} \cdot s_{P}+\lambda_{s} \cdot \eta \cdot Q_{F}+\lambda_{w} \cdot(1-\eta) \cdot Q_{F}-p-c_{P} .
$$

3.3. Optimization Model to Determine the Price of $s_{P}$. In the free-period, the consumer whose utility is positive would adopt $s_{F}$; that is, the consumer who adopts $s_{F}$ in the freeperiod satisfies $v_{i} \geq\left(\left(c_{F}-\left[\lambda_{s} \cdot \eta+\lambda_{w} \cdot(1-\eta)\right] Q_{F}\right) / s_{F}\right)$. The parameter $\Lambda=\lambda_{s} \cdot \eta+\lambda_{w} \cdot(1-\eta)$ is defined as the intensity of the integrated network effect (INEI) cogenerated by the strong and weak relationship groups. The parameter $\Lambda$ is hereafter defined as "Integrated Network Effect Intensity (INEI)."

Given that $0 \leq v_{i} \leq 1$, the demand of $s_{F}$ is $1-\left(\left(c_{F}-\Lambda \cdot Q_{F}\right) / s_{F}\right)$. Inspired by Cheng et al. [16], all consumers of the user base contribute to the generation of network effects. Therefore, $Q_{F}=1-\left(\left(c_{F}-\Lambda \cdot Q_{F}\right) / s_{F}\right)$, and it could be deduced that $Q_{F}=\left(c_{F}-s_{F}\right) /\left(\Lambda-s_{F}\right)$.

In the premium-period, the consumers whose utility is positive would purchase $s_{P}$; that is, the consumer purchasing $s_{P}$ satisfies that $v_{i} \geq\left(p+c_{P}-\Lambda \cdot\left(\left(c_{F}-s_{F}\right) /\left(\Lambda-s_{F}\right)\right)\right) / s_{P}$. Therefore, the number of consumers who purchase $s_{P}$ is $1-\left(\left(p+c_{P}-\Lambda \cdot\left(\left(c_{F}-s_{F}\right) /\left(\Lambda-s_{F}\right)\right)\right) / s_{P}\right)$. The monopolist determines the price of $s_{P}$ to maximize the profit. This decision problem can be expressed by the following optimization model in which $p$ is the decision variable and the 
monopolist's profit $\mathbb{R}$ is the target function. $k_{c}+k_{F} \cdot s_{F}+$ $k_{P} \cdot s_{P}$ is the development cost in which $k_{F}\left(k_{P}\right)$ represents the variable cost of developing $s_{F}\left(s_{P}\right)$ and $k_{c}$ is a constant.

$$
\begin{aligned}
\underset{p}{\operatorname{Max}} \mathbb{R}= & p \cdot\left[1-\frac{p+c_{P}-\Lambda \cdot\left(\left(c_{F}-s_{F}\right) /\left(\Lambda-s_{F}\right)\right)}{s_{P}}\right] \\
& -\left(k_{c}+k_{F} \cdot s_{F}+k_{P} \cdot s_{P}\right) .
\end{aligned}
$$

\section{Results and Analysis}

4.1. The Solution of Optimization Model. The second-order condition of the optimization model (3) is as follows.

$$
\frac{\partial^{2} \mathbb{R}}{\partial p^{2}}=-\frac{2}{s_{P}}
$$

It can be obtained that $\left(\partial^{2} \mathbb{R}\right) /\left(\partial p^{2}\right)<0$, which shows that the optimization model is a concave function and has the maximum value. The optimality of the optimization model (3) can be obtained through the first-order condition that $(\partial \mathbb{R}) /(\partial p)=0$, and the solution of the optimization model (3) is shown as follows.

$$
\begin{aligned}
& p^{*}=\frac{1}{2}\left[s_{P}+c_{P}-\Lambda \cdot\left(\frac{c_{F}-s_{F}}{\Lambda-s_{F}}\right)\right], \\
& \mathbb{R}^{*}=\frac{1}{4 s_{P}}\left[s_{P}+c_{P}-\Lambda \cdot\left(\frac{c_{F}-s_{F}}{\Lambda-s_{F}}\right)\right]^{2}-\left(k_{c}+k_{F} \cdot s_{F}+k_{P} \cdot s_{P}\right) .
\end{aligned}
$$

\subsection{Analysis on the Optimality}

4.2.1. The Situation That $\Lambda$ Is Low. When the intensity of the integrated network effect is low $\left(\Lambda<s_{F} \cdot\left(\left(s_{P}+c_{P}\right) /\right.\right.$ $\left.\left.\left(s_{P}+s_{F}+c_{P}-c_{F}\right)\right)\right), p^{*}<0$ and the following proposition can be obtained.

Proposition 1. When INEI is low, technically, $\Lambda<s_{F}$. $\left(\left(s_{P}+c_{P}\right) /\left(s_{P}+s_{F}+c_{P}-c_{F}\right)\right)$, the provider is supposed to offer the premium product for free and subsidizes the users.

In the other situation that INEI is low, the utility of consumers from the network effect is lower, so as that from the premium product. The lower utility makes less consumers purchase the premium product. In this situation, the vendor could attempt to enlarge the use base through offering cash back services, such as bonus, to consumers.
4.2.2. The Situation That $\Lambda$ Is Moderate. When the intensity of the integrated network effect is moderate $\left(\Lambda=s_{F} \cdot\left(\left(s_{P}+c_{P}\right) /\left(s_{P}+s_{F}+c_{P}-c_{F}\right)\right)\right), p^{*}=0$ and it derives the following proposition.

Proposition 2. When INEI is moderate, technically, $\Lambda=s_{F} \cdot\left(\left(s_{P}+c_{P}\right) /\left(s_{P}+s_{F}+c_{P}-c_{F}\right)\right)$, the provider is supposed to allow the consumers to adopt the premium product freely.

In the situation of INEI being moderate, the utility of consumers obtained from the network effect is still being lower, and this makes the premium product attract less consumers. In this situation, the vendor could attempt to keep the premium product as free to enlarge its demand.

\subsubsection{The Situation That $\Lambda$ Is High. (1) Comparative Static} Analysis on Intensity of Network Effect. When the intensity of the integrated network effect is high $\left(\Lambda>s_{F} \cdot\left(\left(s_{P}+c_{P}\right) /\left(s_{P}+s_{F}+c_{P}-c_{F}\right)\right)\right), p^{*}>0$. Inspired by Ashworth and de Mesquita [19], the features of the optimality could be explored through the monotone comparative static for $p^{*}$ and $\mathbb{R}^{*}$. Therefore, the comparative static analysis on intensity of network effect (i.e., $\lambda_{s}$ and $\lambda_{w}$ ) is employed to investigate the impact of network effect intensity on value-added product price and the vendor's profit. The first-order partial derivatives of $p^{*}\left(\mathbb{R}^{*}\right)$ on $\lambda_{s}\left(\lambda_{w}\right)$ are shown as follows:

$$
\begin{aligned}
& \frac{\partial p^{*}}{\partial \lambda_{s}}=\frac{\partial p^{*}}{\partial \Lambda} \cdot \frac{\partial \Lambda}{\partial \lambda_{s}}=\frac{1}{\Lambda-s_{F}} \cdot \frac{s_{F} \cdot Q_{F}}{2} \cdot \eta, \\
& \frac{\partial p^{*}}{\partial \lambda_{w}}=\frac{\partial p^{*}}{\partial \Lambda} \cdot \frac{\partial \Lambda}{\partial \lambda_{w}}=\frac{1}{\Lambda-s_{F}} \cdot \frac{s_{F} \cdot Q_{F}}{2} \cdot(1-\eta), \\
& \frac{\partial \mathbb{R}^{*}}{\partial \lambda_{s}}=\frac{\partial \mathbb{R}^{*}}{\partial \Lambda} \cdot \frac{\partial \Lambda}{\partial \lambda_{s}}=\frac{1}{\Lambda-s_{F}} \cdot \frac{s_{F} \cdot Q_{F}}{2 s_{P}} \cdot\left(s_{P}+c_{P}-\Lambda \cdot Q_{F}\right) \cdot \eta, \\
& \frac{\partial \mathbb{R}^{*}}{\partial \lambda_{w}}=\frac{\partial \mathbb{R}^{*}}{\partial \Lambda} \cdot \frac{\partial \Lambda}{\partial \lambda_{w}}=\frac{1}{\Lambda-s_{F}} \cdot \frac{s_{F} \cdot Q_{F}}{2 s_{P}} \cdot\left(s_{P}+c_{P}-\Lambda \cdot Q_{F}\right) \cdot(1-\eta),
\end{aligned}
$$

where $Q_{F}$ represents the number of consumers who adopt the free products; thus, $Q_{F} \geq 0 . p^{*}=1 / 2\left[s_{P}+c_{P}-\Lambda \cdot\left(\left(c_{F}-\right.\right.\right.$ $\left.\left.\left.s_{F}\right) /\left(\Lambda-s_{F}\right)\right)\right]$ denotes the optimal price of value-added products; hence, $p^{*} \geq 0, s_{P}+c_{P}-\Lambda \cdot\left(\left(c_{F}-s_{F}\right) /\left(\Lambda-s_{F}\right)\right)$ $\geq 0$. Therefore, whether the first-order partial derivatives are positive or negative is determined by $\Lambda-s_{F}$, and the following equations and proposition are obtained.

$$
\begin{aligned}
& \frac{\partial p^{*}}{\partial \lambda_{s}}\left(\text { or } \frac{\partial p^{*}}{\partial \lambda_{w}}\right) \begin{cases}>0, & \text { if } \Lambda>s_{F}, \\
<0, & \text { if } s_{F} \cdot\left(\left(s_{P}+c_{P}\right) /\left(s_{P}+s_{F}+c_{P}-c_{F}\right)\right)<\Lambda<s_{F},\end{cases} \\
& \frac{\partial \mathbb{R}^{*}}{\partial \lambda_{s}}\left(\text { or } \frac{\partial \mathbb{R}^{*}}{\partial \lambda_{w}}\right) \begin{cases}>0, & \text { if } \Lambda>s_{F}, \\
<0, & \text { if } s_{F} \cdot\left(\left(s_{P}+c_{P}\right) /\left(s_{P}+s_{F}+c_{P}-c_{F}\right)\right)<\Lambda<s_{F} .\end{cases}
\end{aligned}
$$


Proposition 3. When INEI is (or not) sufficiently high, technically, $\Lambda>s_{F} \quad\left(\right.$ or $s_{F} \cdot\left(\left(s_{P}+c_{P}\right) /\left(s_{P}+s_{F}+c_{P}-c_{F}\right)\right)<$ $\left.\Lambda<s_{F}\right)$, the optimal price of the value-added product and the maximum profit of the monopolist increase (or decrease) with the intensity of network effect.

When INEI is sufficiently high, it could be deduced from (2) that the network effects largely increase the consumers' utility of value-added products. In this situation, the consumers are willing to pay a higher price for such items. Therefore, the increasing $\lambda_{s}$ and $\lambda_{w}$ might be helpful to increase the price of the value-added product when INEI is sufficiently high. The increase in prices is beneficial to the profit; hence, $\lambda_{s}$ and $\lambda_{w}$ could also positively impact the profit of the monopoly vendor when it is sufficiently high.

In the other situation that INEI is not sufficiently high, the utility of consumers from the value-added products would be much lower. Hence, the attractiveness of valueadded products to consumers would decline. In this situation, plenty of consumers prefer to use the free product continually in the premium-period rather than purchasing the value-added product, and the monopolist is supposed to decrease the price of the value-added product to lure consumers. Given that the price reduction of value-added products, the revenue of the monopolist would also decrease correspondingly. Therefore, when INEI is not sufficiently high, $\lambda_{s}$ and $\lambda_{w}$ might negatively impact the price of the premium product and the profit of the vendor.

(2) Comparative Static Analysis on Small-World Feature. The comparative static analysis on parameter $\eta$ is performed to explore the impact of the small-world feature on valueadded product price and the monopolist's profit. The firstorder partial derivatives of $p^{*}$ and $\mathbb{R}^{*}$ with $\eta$ are shown as follows:

$$
\begin{aligned}
& \frac{\partial p^{*}}{\partial \eta}=\frac{\partial p^{*}}{\partial \Lambda} \cdot \frac{\partial \Lambda}{\partial \eta}=\frac{1}{\Lambda-s_{F}} \cdot \frac{s_{F} \cdot Q_{F}}{2} \cdot\left(\lambda_{s}-\lambda_{w}\right), \\
& \frac{\partial \mathbb{R}^{*}}{\partial \eta}=\frac{\partial \mathbb{R}^{*}}{\partial \Lambda} \cdot \frac{\partial \Lambda}{\partial \eta}=\frac{1}{\Lambda-s_{F}} \cdot \frac{s_{F} \cdot Q_{F}}{2 s_{P}} \cdot\left(s_{P}+c_{P}-\Lambda \cdot Q_{F}\right) \cdot\left(\lambda_{s}-\lambda_{w}\right),
\end{aligned}
$$

where $Q_{F}$ represents the demand of free products; thus, $Q_{F} \geq 0 . p^{*}=1 / 2\left[s_{P}+c_{P}-\Lambda \cdot\left(\left(c_{F}-s_{F}\right) /\left(\Lambda-s_{F}\right)\right)\right]$ denotes the optimal price of value-added products; hence, $p^{*}>0$, $s_{P}+c_{P}-\Lambda \cdot\left(\left(c_{F}-s_{F}\right) /\left(\Lambda-s_{F}\right)\right)>0$. Since $Q_{F}=\left(\left(c_{F}-s_{F}\right) /\right.$ $\left.\left(\Lambda-s_{F}\right)\right), s_{P}+c_{P}-\Lambda \cdot Q_{F}>0$. Therefore, whether the firstorder partial derivatives are positive or negative is determined by $\Lambda-s_{F}$. Since $\Lambda-s_{F}=\lambda_{s} \cdot \eta+\lambda_{w} \cdot(1-\eta)-s_{F}$, the following equations and proposition are obtained.

$$
\frac{\partial p^{*}}{\partial \eta}\left(\text { or } \frac{\partial \mathbb{R}^{*}}{\partial \eta}\right) \begin{cases}>0, & \text { if } \eta>\left(\left(s_{F}-\lambda_{w}\right) /\left(\lambda_{s}-\lambda_{w}\right)\right), \\ <0, & \text { if } \eta<\left(\left(s_{F}-\lambda_{w}\right) /\left(\lambda_{s}-\lambda_{w}\right)\right) .\end{cases}
$$

Proposition 4. When the small-world feature of the customer group is (or not) sufficiently prominent, technically, $\eta<\left(\left(s_{F}-\lambda_{w}\right) /\left(\lambda_{s}-\lambda_{w}\right)\right)$ (or $\left.\eta>\left(\left(s_{F}-\lambda_{w}\right) /\left(\lambda_{s}-\lambda_{w}\right)\right)\right)$, the optimal price of value-added products and the maximum profit of the vendor decrease (or increase) with the smallworld feature.

When the small-world feature of consumers is sufficiently prominent, the value of the small-world parameter $\eta$ bellows the threshold $\left(s_{F}-\lambda_{w}\right) /\left(\lambda_{s}-\lambda_{w}\right)$. Thus, the size of the consumer's strong relationship group is small, and the network effect generated by the user group is weak. In this situation, the increasing value of $\eta$ does not substantially improve the consumers' utility from value-added products. Therefore, the monopolist is supposed to decrease price to attract consumers. The decrease of price is not beneficial to the revenue of the firm; hence, the small-world coefficiency $\eta$ negatively impacts $\mathbb{R}^{*}$ if its value is not sufficiently high.

When the small-world feature of the consumer group is not sufficiently prominent, the value of $\eta$ exceeds the threshold $\left(s_{F}-\lambda_{w}\right) /\left(\lambda_{s}-\lambda_{w}\right)$. Hence, the consumer's strong relationship group is large. According to (2), the consumers who are affected by the strong network effect would obtain a higher utility when using value-added products; and those consumers would be willing to pay a higher price for the value-added product. In this situation, the small-world coefficiency $\eta$ positively impacts $p^{*}$, that is, $p^{*}$ increases with $\eta$, and vice versa. The increase in prices is beneficial to the vendor's profit; hence, $\eta$ performs a positive impact on $\mathbb{R}^{*}$.

4.3. Managerial Insights. The models and the propositions presented in the current work have important implications for the digital products firms in terms of management and operation.

First, the network effect might exert increasingly complicated impacts on the price of products and the profits of firms due to the small-world feature of the user base. Specifically, the network effect would not impose the positive impact until the intensity of the integrated network effect is high or the small-world feature of the consumer group is not prominent. Therefore, digital products firms are supposed to increase their profits through increasing the impact of each consumer (to other consumers) in the user base or make the consumers maintain close contact with as many other individuals as possible, through which the consumers would obtain considerable positive externalities from their social group.

Second, the digital products firms are supposed to set skimming prices for their products in the situation that the intensity of the integrated network effect is sufficiently high, or the size of the consumer's strong relationship group is large. In this situation, consumers can obtain high utility from the value-added products via the smallworld network and, thus, are willing to pay a high price for the value-added product. In such a market environment, the firms could obtain profit fast through the high price.

Third, the digital products firms should adopt penetration pricing strategy in the situation that the intensity of the integrated network effect is not sufficiently high or the size of consumer's strong relationship group is small. In this situation, the network effect has a negative effect on the 
consumer utility and then the consumers are not willing to pay a high price for the value-added products. In such a market environment, the firms should attract consumers by providing free products and then expand the market size of value-added products through the low price. Moreover, if integrated network effect becomes much lower, the firms are supposed to enlarge their installed base through complete strategy (the premium product is also free) or even offering cash back bonus to the consumers.

\section{Conclusions}

A significant reason of the global soar in digital products is the popularity of the Freemium business model; and the current work explores the effect of the user base's smallworld feature in the Freemium of digital products. In this work, the consumer utility model is presented, and the model considers the small-world network feature. On the basis of the utility model, the optimization model is derived in which the small-world feature is a key parameter. By solving the optimization model and conducting comparative static analyses, the current work investigates the mechanism of the small-world network effect on the price of value-added products and the profit of digital products vendors. The results of the comparative statics show that taking the smallworld feature into account, the network externality would generate positive effect on the premium product's price and the vendors' profit only if the small-world feature of the user base is not prominent or the intensity of network externality is sufficiently high. The current work shows the great significance of the network effect; since the definite impact of the network effect to firms' profit, the digital products providers in the reality, such as Facebook, YouTube, and Twitter, are struggling to enlarge their user base.

Finally, the current work still has some limitations. First, the current paper leaves out another form of Freemium. For example, the digital products firms could sell advertising space within the free product but charge to the firms who intend to advertise in the space $[20,21]$. The future work might explore the left forms of Freemium to provide a comprehensive understanding of this popular business model in digital products industry. Second, the current work does not take into account that the utility of consumers may decrease with time. Existing literatures, such as work by Shivendu and Zhang [22] and Dou et al. [23], have analyzed the effects of decreasing consumers' utility on the firms' determinations. It would be interesting, inspired by the existing literatures, to investigate the Freemium model with the variable utility. Third, the Freemium model is also widely adopted by the digital products firms providing novel digital services. For instance, the Software-as-a-Service (SaaS) firms provide the flexible on-demand software service for their consumers [24]. It is definitely a challenge exploring the Freemium model from the perspective of these new forms of digital services.

\section{Data Availability}

The data used to support the findings of this study are available from the corresponding author upon request.

\section{Conflicts of Interest}

The author has no conflicts of interest.

\section{Acknowledgments}

The current paper was supported by (1) Hebei Provincial Department of Education (SQ191078); (2) Research Foundation Program of Hebei University of Economics and Business (2017KYY01; 2018PY08; and 2019JYQ02); and (3) Key Research Institute of Humanities and Social Science at Universities of Hebei Province.

\section{References}

[1] F. Wilson, "Freemium business model," 2006, http://www.avc. com/a_vc/2006/03/the_freemium_bu.html.

[2] C. Anderson, Free: The Future of a Radical Price, Hyperion, Santa clara, CA, USA, 2009.

[3] G. Oestreicher-Singer and L. Zalmanson, "Content or community? a digital business strategy for content providers in the social age," MIS Quarterly, vol. 37, no. 2, pp. 591-616, 2013.

[4] C.-L. Hsu and J. C.-C. Lin, "What drives purchase intention for paid mobile apps? - an expectation confirmation model with perceived value," Electronic Commerce Research and Applications, vol. 14, no. 1, pp. 46-57, 2015.

[5] C. Z. Liu, Y. A. Au, and H. S. Choi, "Effects of freemium strategy in the mobile app market: an empirical study of google play," Journal of Management Information Systems, vol. 31, no. 3, pp. 326-354, 2014.

[6] X. Gu, P. K. Kannan, and L. Ma, How Companies Can Get the Most Out of a Freemium Business Model, Harvard Business Review, Brighton, MA, USA, 2019, https://hbr.org/2019/03/ how-companies-can-get-the-most-out-of-a-freemiumbusiness-model.

[7] A. B. Holm and F. Günzel-Jensen, "Succeeding with freemium: strategies for implementation," Journal of Business Strategy, vol. 38, no. 2, pp. 16-24, 2017.

[8] G. P. Cziehso, T. Schaefers, and M. Kukar-Kinney, "Free no more-investigating customer reactions to unexpected freeto-fee switches," Journal of Business Research, vol. 101, pp. 229-242, 2019.

[9] J. D. Shulman and X. Geng, "Does it pay to shroud in-app purchase prices?" Information Systems Research, vol. 30, no. 3, pp. 856-871, 2019.

[10] J.-w. Jang, J. Woo, A. Mohaisen, J. Yun, and H. K. Kim, "Malnetminer: malware classification approach based on social network analysis of system call graph," Mathematical Problems in Engineering, vol. 2015, Article ID 769624, pp. 1-20, 2015.

[11] A. Prasad, R. Venkatesh, and V. Mahajan, "Optimal bundling of technological products with network externality," Management Science, vol. 56, no. 12, pp. 2224-2236, 2010.

[12] M. F. Niculescu and D. J. Wu, "Economics of free under perpetual licensing: implications for the software industry," Information Systems Research, vol. 25, no. 1, pp. 173-199, 2014.

[13] R. Bapna and A. Umyarov, "Do your online friends make you pay? a randomized field experiment on peer influence in online social networks," Management Science, vol. 61, no. 8, pp. 1902-1920, 2015. 
[14] D. J. Watts and S. H. Strogatz, "Collective dynamics of "smallworld” networks," Nature, vol. 393, no. 6684, pp. 440-442, 1998.

[15] J. Hamari, N. Hanner, and J. Koivisto, "Service quality explains why people use freemium services but not if they go premium: an empirical study in free-to-play games," International Journal of Information Management, vol. 37, no. 1, pp. 1449-1459, 2017.

[16] H. K. Cheng, S. Li, and Y. Liu, "Optimal software free trial strategy: limited version, time-locked, or hybrid?” Production and Operations Management, vol. 24, no. 3, pp. 504-517, 2015.

[17] Z. Shi, K. Zhang, and K. Srinivasan, "Freemium as an optimal strategy for market dominant firms," Marketing Science, vol. 38, no. 1, pp. 150-169, 2019.

[18] D. J. Watts, Six Degrees: The Science of a Connected Age, WW Norton, Newyork, NY, USA, 2004.

[19] S. Ashworth and E. B. de Mesquita, "Monotone comparative statics for models of politics," American Journal of Political Science, vol. 50, no. 1, pp. 214-231, 2006.

[20] G. Appel, B. Liba, i. E. Muller, and R. Shachar, "On the monetization of mobile apps," International Journal of Research in Marketing, 2019, In press.

[21] S. Sato, "Freemium as optimal menu pricing," International Journal of Industrial Organization, vol. 63, pp. 480-510, 2019.

[22] S. Shivendu and Z. Zhang, "Versioning in the software industry: heterogeneous disutility from underprovisioning of functionality," Information Systems Research, vol. 26, no. 4, pp. 731-753, 2015.

[23] Y. Dou, Y. J. Hu, and D. J. Wu, "Selling or leasing? pricing information goods with depreciation of consumer valuation," Information Systems Research, vol. 28, no. 3, pp. 585-602, 2017.

[24] Y. Wei, D. Kudenko, S. Liu, L. Pan, L. Wu, and X. Meng, "A reinforcement learning based auto-scaling approach for SaaS providers in dynamic cloud environment," Mathematical Problems in Engineering, vol. 2019, Article ID 5080647, pp. 1-11, 2019. 